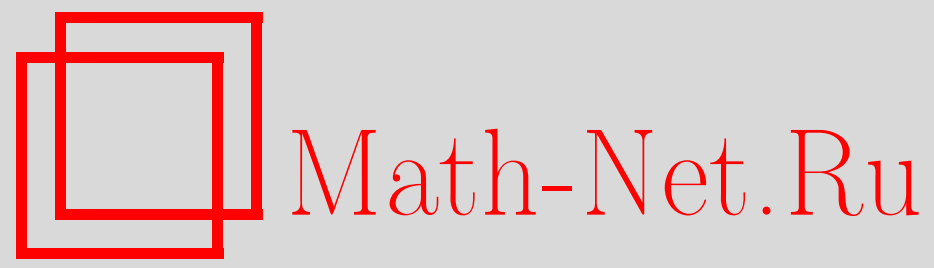

К. Д. Жуков, Об обобщении метода Дюжелла, Матем. вопр. криптогр., 2013, том 4, выпуск 3, 7-19

DOI: https://doi.org/10.4213/mvk90

Использование Общероссийского математического портала Math-Net.Ru подразумевает, что вы прочитали и согласны с пользовательским соглашением

http://www.mathnet.ru/rus/agreement

Параметры загрузки:

IP: 54.92.164.108

26 апреля 2023 г., 10:18:37 
УДК: $511.53+519.719 .2$

\title{
Об обобщении метода Дюжелла
}

\author{
к. Д. Жуков \\ Лаборатория ТВП, Москва
}

Получено 20.IV.2012

В существующих реализациях криптосистемы RSA с модулем $N=p q$, как правило, используются большие секретные экспоненты. Тем не менее известно много теоретических результатов по криптоанализу системы RSA с малой секретной экспонентой. Метод Дюжелла восстанавливает секретные экспоненты $d<D N^{0.25}$ с трудоемкостью $O(D \ln D)$ арифметических операций и затратами памяти $O(D)$. Де Вегер предложил атаковать секретные экспоненты $d<\frac{N^{0.75}}{p-q}$. В работе описывается обобщение метода Дюжелла, позволяющее с трудоемкостью $O(D \ln D)$ арифметических операций и памятью $O(D)$ атаковать экспоненты $d<D \frac{N^{0.75}}{p-q}$.

Ключевые слова: криптосистема RSA, диофантовы приближения, метод «встреча посередине»

\section{On a generalization of the Dujella method}

\section{K. D.Zhukov}

\section{TPA Laboratory, Moscow}

Abstract. As a rule, large secret exponents are used in practical realizations of RSA cryptosystem with modulus $N=p q$. Nevertheless, there are many theoretical results on the cryptanalysis of RSA system with a small secret exponent. A method suggested by Dujella recovers secret exponents $d<D N^{0.25}$ with a run-time complexity $O(D \ln D)$ and space complexity $O(D)$. Weger have suggested an attack on the secret exponents $d<\frac{N^{0.75}}{p-q}$. We describe a generalization of the Dujella method to attack the exponents $d<D \frac{N^{0.75}}{p-q}$ with run-time complexity $O(D \ln D)$ and space complexity $O(D)$.

Key words: RSA cryptosystem, Diophantine approximations, meet-in-the-middle attack

Citation: Mathematical Aspects of Cryptography, 2013, vol. 4, no. 3, pp. $7-19$ (Russian). 


\section{1. Введение}

Криптографическая система RSA является системой с открытым ключом. В настоящее время система RSA широко распространена и повсеместно используется в сети Интернет.

Каждый абонент, использующий систему RSA, имеет открытый и секретный ключи. Открытый ключ - это пара чисел $(e, N)$. Натуральное число $N$ является произведением двух больших простых чисел $p$ и $q$. Натуральное число $е$ удовлетворяет условию НОД( $(e, \phi(N))=1$, где $\phi-$ функция Эйлера. Секретным ключом является натуральное число $d$, определяемое условием $e d \equiv 1(\bmod \phi(N))$. Числа $e$ и $d$ называются открытой и секретной экспонентами, а число $N$ - модулем шифрования. Для зашифрования открытый текст $x$, рассматриваемый как натуральное число в интервале от 0 до $N-1$, возводится в степень $e$ по модулю $N: y=x^{e} \bmod N$. Последнее равенство понимается как равенство в кольце $\mathbb{Z} / N \mathbb{Z}$, при этом наименьший неотрицательный вычет класса отождествляется со всем классом. Расшифрование проводится возведением в степень $d$ по модулю $N: x=y^{d} \bmod N$.

Простые числа $p$ и $q$ необходимо хранить в секрете, так как, зная их, противник может вычислить $d$ с помощью расширенного алгоритма Евклида. Таким образом, стойкость системы RSA основана на проблеме факторизации.

Существует ряд условий на параметры системы RSA, при которых она является нестойкой. Такие параметры называются слабыми. Одной из известных слабостей является слишком малая секретная экспонента $d$. В настоящее время теоретически самой сильной полиномиальной атакой на малую секретную экспоненту является атака Боне и Дюрфи [5]. Атака основывается на методе Копперсмита и позволяет восстанавливать значение $d<N^{0.292}$. Существует класс атак на малую секретную экспоненту $d$, основанных на аппарате непрерывных дробей. Это, например, атака Винера и ее различные модификации и обобщения (атаки Хинека, Дюжелла и де Вегера).

Атаки, основанные на вычислении цепных дробей, очень быстры, но границы их применимости существенно уже. Например, атака Винера восстанавливает экспоненту $d<\frac{1}{3} N^{0.25}$, если $p$ и $q$ имеют одинаковый размер в битах. Однако с помощью перебора множества мощности $O\left(D^{2}\right)$ границу применимости атаки можно расширить до $d<D N^{0.25}$. Такие атаки предложены в $[7,8]$. В работе [9] Дюжелла предложил использовать метод «встреча посередине» [4] для перебора множества мощности $O\left(D^{2}\right)$. Тем самым трудоемкость метода была понижена до $O(D \ln D)$ арифметических операций с затратами памяти $O(D)$, что позволило расширить границы применимости атаки. В таком виде атака оказалась сопоставимой с атакой Боне и Дюрфи. 
Другой возможной слабостью системы RSA является использование слишком близких значений $p$ и $q$. Если разность $p-q$ имеет порядок $N^{0.25}$, то эффективным оказывается метод факторизации Ферма. Де Вегер [10] предложил обобщение атаки Винера на случай, когда разность $p-q$ и число $d$ малы. Причем, чем ближе $p$ и $q$, тем большие значения $d$ оказываются слабыми. А именно, если разность $p-q$ не превышает $N^{\beta}$, то атака позволяет восстанавливать секретные экспоненты $d<N^{0.75-\beta}$. Также в работе [10] обобщается атака Боне и Дюрфи на случай, когда $p$ и $q$ близки.

В данной работе описывается новая атака на криптосистему RSA с малой секретной экспонентой и малой разностью простых сомножителей модуля. Атака объединяет идеи Дюжелла и де Вегера. Если известно, что разность $p-q$ не превышает $N^{\beta}$, то новый метод позволяет восстанавливает секретную экспоненту $d$, не превосходящую $D N^{0.75-\beta}$, с трудоемкостью $O(D \ln D)$ арифметических операций и затратами памяти $O(D)$.

Дальнейшее изложение построено следующим образом. В части 2 приводятся основные определения теории цепных дробей и описывается атака Винера с модификацией де Вегера. В части 3 описывается атака Дюжелла. В части 4 обосновывается новая атака. Далее приводятся результаты экспериментов с программными реализациями атаки Дюжелла и ее новым обобщением. В конце приводится сравнение с предложенным де Вегером обобщением атаки Боне и Дюрфи.

\section{2. Метод Винера}

Напомним основные определения согласно [1]. Бесконечной цепной дробью называется выражение вида

$$
a_{0}+\frac{1}{a_{1}+\frac{1}{a_{2}+\ldots}}
$$

где число $a_{0}$ - целое, а числа $a_{n}, n=1,2, \ldots$ - натуральные.

Выражение (1) записывают как $\left\langle a_{0}, a_{1}, a_{2}, \ldots\right\rangle$. Подходящей дробью $\frac{P_{n}}{Q_{n}}$ к бесконечной цепной дроби (1) называется конечная цепная дробь

$$
\frac{P_{n}}{Q_{n}}=\left\langle a_{0}, a_{1}, a_{2}, \ldots, a_{n}\right\rangle .
$$

Известно, что любое действительное число $\alpha$ представляется в виде цепной дроби. Подходящие дроби можно рассматривать как приближения к числу $\alpha$. Существует алгоритм, позволяющий рекуррентно находить подходящие дроби для заданного действительного числа. Атака Винера основывается на следующей теореме. 
Теорема 1 ([1, теорема 245]). Пусть дано действительное число $\alpha$ и ненулевые взаимно простые целье числа а и $b$, такие что $\left|\alpha-\frac{a}{b}\right|<\frac{1}{2 b^{2}}$. Тогда $\frac{a}{b}$ - одна из походящих дробей $\kappa \alpha$.

Теорема 1 говорит, что если рациональная дробь в некотором смысле хорошо аппроксимирует действительное число, то она является его подходящей дробью. Винер применил эту теорему к криптосистеме RSA следующим образом.

Для криптосистемы RSA справедливо равенство $\frac{e}{\phi(N)}-\frac{k}{d}=\frac{1}{\phi(N) d}$, где целое $k$ определяется условием $e d=1+k \phi(N)$. Отсюда следует, что дробь $\frac{k}{d}$ является хорошим приближением к числу $\frac{e}{\phi(N)}$, а последнее при некоторых условиях аппроксимируется числом $\frac{e}{N}$. Тогда дробь $\frac{k}{d}$ хорошо приближает число $\frac{e}{N}$. Несложно показать, что если $d<\frac{1}{3} N^{0.25}$ и двоичные представления $p$ и $q$ имеют одинаковую длину, то выполняется неравенство

$$
\left|\frac{e}{N}-\frac{k}{d}\right|<\frac{1}{2 d^{2}} .
$$

$\mathrm{B}$ этом случае мы можем найти секретную экспоненту $d$ среди знаменателей подходящих дробей к известному числу $\frac{e}{N}$.

Де Вегер предложил аппроксимировать неизвестное $\phi(N)=(p-1)(q-$ $-1)$ числом $\left(N^{0.5}-1\right)^{2}$ вместо $N$. Очевидно, что чем ближе значения $p$ и $q$, тем лучше становится выбранная аппроксимация. Де Вегер в [10] показал, что если разность $p-q$ не превышает $N^{\beta}$, то неравенство

$$
\left|\frac{e}{\left(N^{0.5}-1\right)^{2}}-\frac{k}{d}\right|<\frac{1}{2 d^{2}}
$$

выполняется при $d<N^{0.75-\beta}$.

\section{3. Метод Дюжелла}

Верхул и ван Тилборг [7] предложили искать дробь $\frac{k}{d}$ среди дробей вида $\frac{r P_{m+1}+s P_{m}}{r Q_{m+1}+s Q_{m}}$, где $\frac{P_{i}}{Q_{i}}-i$-я подходящая дробь для числа $\frac{e}{N}$. Так как определитель системы

$$
\begin{aligned}
r P_{m+1}+s P_{m} & =k, \\
r Q_{m+1}+s Q_{m} & =d
\end{aligned}
$$

всегда обратим, $P_{m+1} Q_{m}-Q_{m+1} P_{m}=(-1)^{m}$, то для любого $m$ существуют такие целые $r$ и $s$, что выполняется равенство $\frac{k}{d}=\frac{r P_{m+1}+s P_{m}}{r Q_{m+1}+s Q_{m}}$. Дюжелла [8], 
переформулировав результат Верхула и ван Тилборга, указал такое $m$, что для чисел $r$ и $s$ выполняются следующие оценки:

$$
\begin{aligned}
& r<\max \left\{\sqrt{2.122\left(a_{m+3}+2\right)}\left(a_{m+2}+1\right) D, \sqrt{2.122\left(a_{m+2}+2\right)} D\right\}, \\
& s<\max \left\{2 \sqrt{2.122\left(a_{m+3}+2\right)} D, \sqrt{2.122\left(a_{m+2}+2\right)}\left(a_{m+1}+1\right) D\right\},
\end{aligned}
$$

где $D=d / N^{0.25}$. В работе [7] экспериментально установлено, что с большой вероятностью числа $r$ и $s$ не превосходят $16 D$.

Дюжелла в работе [8] получил новые оценки для чисел $r$ и $s$ в случае, когда дробь $\frac{k}{d}$ ищется среди дробей вида $\frac{r P_{m+1}+s P_{m}}{r Q_{m+1}+s Q_{m}}, \frac{r P_{m+2}-s P_{m+1}}{r Q_{m+2}-s Q_{m+1}}$ и $\frac{r P_{m+3}+s P_{m+2}}{r Q_{m+3}+s Q_{m+2}}$ для специально выбранного $m$. В [9] экспериментально установлено, что с вероятностью $98 \%$ числа $r$ и $s$ не превосходят $4 D$. Дюжелла вывел свои оценки с помощью неравенств

$$
\frac{2 e}{N \sqrt{N}}<\frac{k}{d}-\frac{e}{N}<\frac{2.122 e}{N \sqrt{N}}<\frac{2.122 D^{2}}{d^{2}}
$$

и доказанной им теоремы о диофантовых приближениях.

Теорема 2 ([8, теорема 1]). Пусть дано действительное число $\alpha$ и ненулевые взаимно простые целые числа а и $b$, такие что $\left|\alpha-\frac{a}{b}\right|<\frac{c}{b^{2}}$, где с - некоторая вещественная положительная константа. Тогда $(a, b)=$ $=\left(r P_{m+1} \pm s P_{m}, r Q_{m+1} \pm s Q_{m}\right)$ для некоторых неотрицательных иелых $r, s$ u m, удовлетворяюших условию $r s<2 c$.

Дюжелла оценил числа $r$ и $s$, исходя из следующих предпосылок. Пусть $m$ - максимальный нечетный номер подходящей дроби к числу $\frac{e}{N}$, для которой выполняется неравенство $\frac{P_{m}}{Q_{m}}-\frac{e}{N}>\frac{2.122 e}{N \sqrt{N}}$. Возможны два случая, зависящие от того, больше или меньше подходящая дробь $\frac{P_{m+2}}{Q_{m+2}}$ числа $\frac{k}{d}$. Предположим, что $\frac{P_{m+2}}{Q_{m+2}} \geq \frac{k}{d}$. В работе [8] с помощью неравенств (6) показано, что если в этом случае мы будем искать дробь $\frac{k}{d}$ в виде $\frac{r^{\prime} P_{m+3}+s^{\prime} P_{m+2}}{r^{\prime} Q_{m+3}+s^{\prime} Q_{m+2}}$, то числа $r^{\prime}$ и $s^{\prime}$ не будут превосходить величин $\frac{0.061 \sqrt{2.122\left(a_{m+3}+2\right)}}{a_{m+3}} D$ и $\sqrt{2.122\left(a_{m+3}+2\right)} D$ соответственно.

Предположим теперь, что $\frac{P_{m+2}}{Q_{m+2}}<\frac{k}{d}$. Пусть мы ищем дробь $\frac{k}{d}$ в виде $\frac{r P_{m+1}+s P_{m}}{r Q_{m+1}+s Q_{m}}$ или $\frac{s P_{m+2}-t P_{m+1}}{s Q_{m+2}-t Q_{m+1}}$. Используя правые части неравенств (6) и теорему 2, Дюжелла показал, что

$$
r<2.061 \sqrt{a_{m+2}} D, \quad t<2.061 \sqrt{a_{m+2}} D,
$$

а для $s$ по-прежнему справедлива оценка (5). 
Далее Дюжелла предложил применить метод «встреча посередине» для опробования чисел $r$ и $s$. Каждой паре соответствует опробуемое значение секретной экспоненты $d$, истинность которого проверяется условием $\left(2^{e}\right)^{d} \equiv 2(\bmod N)$. Пусть, например, секретная экспонента ищется в виде $d=r Q_{m+1}+s Q_{m}$. Тогда отбраковывать пары $r, s$ можно, исходя из условия

$$
2^{e\left(r Q_{m+1}+s Q_{m}\right)} \equiv 2 \quad(\bmod N) .
$$

В кольце вычетов по модулю $N$ можно заранее вычислить элементы $a=$ $=2^{e Q_{m+1}} \bmod N$ и $b=\left(2^{e Q_{m}}\right)^{-1} \bmod N$ для фиксированного $m$ и, перебирая $r$ и $s$, проверять выполнение условия

$$
a^{r} \equiv 2 b^{s} \quad(\bmod N) .
$$

Такой критерий позволяет применить метод «встреча посередине». Для всех опробуемых значений $r$ составляется таблица элементов $a^{r} \bmod N$. Затем для каждого опробуемого $s$ в таблице ищется элемент $2 b^{s} \bmod N$. При $r$ и $s$ порядка $O(D)$ трудоемкость такого метода составляет $O(D \ln D)$ арифметических операций при затратах памяти $O(D)$.

\section{4. Обобщение атаки Дюжелла}

Описанную в части 3 атаку можно обобщить на случай, когда известно, что разность $p-q$ и экспонента $d$ не превосходят заданных значений. Для этого вместо неравенств (6) будем использовать неравенства

$$
0<\frac{k}{d}-\frac{e}{\left(N^{0.5}-1\right)^{2}}<\frac{N^{1-2 \lambda}+4}{3 N}<\frac{5 D^{2}}{3 d^{2}},
$$

где действительные положительные величины $\lambda<0.5$ и $D>1$ определяются условием

$$
\frac{d}{D}<N^{\lambda}<\frac{N^{0.75}}{p-q} .
$$

Справедливость неравенств (7) при некоторых дополнительных требованиях вытекает из следующих двух лемм.

Лемма 1. Пусть модуль $N=p q$, где $p-q>2 N^{0.25}$. Пусть $k$, определяемое условием еd $=1+k \phi(N)$, не равно 1. Тогда

$$
\frac{k}{d}-\frac{e}{N-2 N^{0.5}+1}>0 .
$$


Доказательство. Несложно показать, что если для некотого числа $c$ разность чисел $p$ и $q$ больше $2 c$, то $p>N^{0.5}+c$. Тогда при $c=N^{0.25}$ сумму $p$ и $q$ можно оценить снизу:

$$
p+q=p+\frac{N}{p}>2 N^{0.5}+\frac{1}{1+N^{-0.25}} .
$$

Преобразуем разность из утверждения леммы следующим образом:

$$
\begin{aligned}
\frac{k}{d}-\frac{e}{N-2 N^{0.5}+1} & =\frac{k\left(N-2 N^{0.5}+1\right)-1-k(N-p-q+1)}{d\left(N-2 N^{0.5}+1\right)}= \\
& =\frac{k\left(p+q-2 N^{0.5}\right)-1}{d\left(N-2 N^{0.5}+1\right)} .
\end{aligned}
$$

Из (9) и условия $k \geq 2$ следует, что

$$
k\left(p+q-2 N^{0.5}\right)>k \frac{1}{1+N^{-0.25}}>1 .
$$

Отсюда следует, что $k\left(p+q-2 N^{0.5}\right)-1>0$, и лемма доказана.

Лемма 2. Пусть модуль $N=p q u \phi(N)>\frac{3}{4} N$. Пусть секретная экспонента d удовлетворяет условию (8) при некоторых действительных положительных $\lambda<0.5$ и $D>1$. Тогда выполняется двойное неравенство

$$
\left|\frac{e}{N+1-2 N^{0.5}}-\frac{k}{d}\right|<\frac{N^{1-2 \lambda}+4}{3 N}<\frac{5 D^{2}}{3 d^{2}} .
$$

Доказательство. С помощью несложных преобразований получаем цепочку неравенств:

$$
\begin{aligned}
& \left|\frac{e}{N+1-2 N^{0.5}}-\frac{k}{d}\right| \leq e\left|\frac{1}{N+1-2 N^{0.5}}-\frac{1}{\varphi(N)}\right|+\left|\frac{e}{\varphi(N)}-\frac{k}{d}\right|< \\
& <\varphi(N) \frac{\left|\left(N+1-2 N^{0.5}\right)-\varphi(N)\right|}{N+1-2 N^{0.5}}+\frac{1}{\varphi(N) d}<\frac{1}{\varphi(N)}\left(\frac{(p-q)^{2}}{4 N^{0.5}}+\frac{1}{d}\right) .
\end{aligned}
$$

Так как $\phi(N)>\frac{3}{4} N$ и $N^{\lambda}<\frac{N^{0.75}}{p-q}$, то

$$
\begin{gathered}
\left|\frac{e}{N+1-2 N^{0.5}}-\frac{k}{d}\right|<\frac{4}{3}\left(\frac{(p-q)^{2}}{4 N^{1.5}}+\frac{1}{N d}\right)< \\
<\frac{4}{3}\left(\frac{1}{4 N^{2 \lambda}}+\frac{1}{N}\right)=\frac{N^{1-2 \lambda}+4}{3 N}
\end{gathered}
$$

2013, T. 4, № 3, C. 7-19 
и левое неравенство леммы доказано. С другой стороны, из условий $\frac{d}{D}<N^{\lambda}$ и $\lambda<0.5$ следует, что

$$
\frac{4}{3}\left(\frac{1}{4 N^{2 \lambda}}+\frac{1}{N}\right)<\frac{4}{3}\left(\frac{1}{4 N^{2 \lambda}}+\frac{1}{N^{2 \lambda}}\right)=\frac{4}{3} \cdot \frac{5}{4 N^{2 \lambda}}<\frac{5 D^{2}}{3 d^{2}},
$$

и правое неравенство леммы доказано.

Получим теперь оценки для чисел $r^{\prime}, s^{\prime}, r, s$ и $t$ аналогично работе [8]. Зафиксируем последнее нечетное $m$, для которого еще выполняется неравенство $\frac{P_{m}}{Q_{m}}-\frac{e}{N+1-2 N^{0.5}}>\frac{N^{1-2 \lambda}+4}{3 N}$. Вначале, как и в [8], оценим величину $\frac{1}{Q_{m+2}}$. Для этого воспользуемся известным неравенством для подходящих дробей к $\alpha$ (см. [2, теорема 13])

$$
\frac{1}{Q_{m}\left(Q_{m+1}+Q_{m}\right)}<\left|\alpha-\frac{P_{m}}{Q_{m}}\right| .
$$

Тогда из него и леммы 2 по выбору $m$ вытекает

$$
\frac{1}{Q_{m+2}\left(Q_{m+3}+Q_{m+2}\right)}<\frac{5 D^{2}}{3 d^{2}} .
$$

Преобразуя левую часть, получаем

$$
\frac{1}{Q_{m+2}^{2}\left(a_{m+3}+1+\frac{Q_{m+1}}{Q_{m+2}}\right)}<\frac{5 D^{2}}{3 d^{2}},
$$

откуда следует, что

$$
\frac{1}{Q_{m+2}}<\frac{D}{d} \sqrt{\frac{5}{3}\left(a_{m+3}+2\right)}
$$

Пусть $\frac{P_{m+2}}{Q_{m+2}} \geq \frac{k}{d}$. Тогда ищем дробь $\frac{k}{d}$ в виде $\frac{r^{\prime} P_{m+3}+s^{\prime} P_{m+2}}{r^{\prime} Q_{m+3}+s^{\prime} Q_{m+2}}$. При этом

$$
\begin{gathered}
r^{\prime}=d Q_{m+2}\left(\frac{P_{m+2}}{Q_{m+2}}-\frac{k}{d}\right)<d Q_{m+2}\left(\frac{P_{m+2}}{Q_{m+2}}-\frac{e}{\left(N^{0.5}-1\right)^{2}}\right)< \\
<d Q_{m+2} \cdot \frac{1}{Q_{m+2} Q_{m+3}}=\frac{d}{Q_{m+3}}<\frac{d}{Q_{m+2}}<D \sqrt{\frac{5}{3}\left(a_{m+3}+2\right) .}
\end{gathered}
$$

Аналогично,

$$
\begin{gathered}
s^{\prime}=d Q_{m+3}\left(\frac{k}{d}-\frac{P_{m+3}}{Q_{m+3}}\right)<d Q_{m+3}\left(\frac{P_{m+2}}{Q_{m+2}}-\frac{P_{m+3}}{Q_{m+3}}\right)= \\
=\frac{d}{Q_{m+2}}<D \sqrt{\frac{5}{3}\left(a_{m+3}+2\right) .}
\end{gathered}
$$


Если же $\frac{P_{m+2}}{Q_{m+2}}<\frac{k}{d}$, то воспользуемся неравенством $\left|\frac{k}{d}-\frac{e}{\left(N^{0.5}-1\right)^{2}}\right|<\frac{5 D^{2}}{3 d^{2}}$. Последнее позволяет применить теорему 2. Из деталей ее доказательства [8, теорема 1] следует, что если $\frac{k}{d}=\frac{r P_{m+1}+s P_{m}}{r Q_{m+1}+s Q_{m}}$ или $\frac{k}{d}=\frac{s P_{m+2}-t P_{m+1}}{s Q_{m+2}-t Q_{m+1}}$, то $r s<\frac{10}{3} D^{2}$ и $s t<\frac{10}{3} D^{2}$. Более того, из ее доказательства следует, что $r<a_{m+2} s$ и $t<a_{m+2} s$. Поэтому $r<\sqrt{\frac{10}{3} a_{m+2}} D$ и $t<\sqrt{\frac{10}{3} a_{m+2}} D$. Оценка величины $s$ зависит от знака числа $\frac{e}{\left(N^{0.5}-1\right)^{2}}-\frac{P_{m+1}}{Q_{m+1}}-\frac{N^{1-2 \lambda}+4}{3 N}$. Пусть это число положительно, тогда

$$
\begin{aligned}
s=d Q_{m+1}\left(\frac{k}{d}-\frac{P_{m+1}}{Q_{m+1}}\right) & <2 d Q_{m+1}\left(\frac{e}{\left(N^{0.5}-1\right)^{2}}-\frac{P_{m+1}}{Q_{m+1}}\right)<\frac{2 d}{Q_{m+2}}< \\
& <2 D \sqrt{\frac{5}{3}\left(a_{m+3}+2\right) .}
\end{aligned}
$$

Если же $\frac{e}{\left(N^{0.5}-1\right)^{2}}-\frac{P_{m+1}}{Q_{m+1}} \leq \frac{N^{1-2 \lambda}+4}{3 N}$, то

$$
\begin{gathered}
s=d Q_{m+1}\left(\frac{k}{d}-\frac{P_{m+1}}{Q_{m+1}}\right)<d Q_{m+1}\left(\frac{P_{m}}{Q_{m}}-\frac{P_{m+1}}{Q_{m+1}}\right)=\frac{d}{Q_{m}}< \\
<\frac{d\left(a_{m+1}+1\right)}{Q_{m+1}}<D \sqrt{\frac{5}{3}\left(a_{m+2}+2\right)\left(a_{m+1}+1\right) .}
\end{gathered}
$$

Таким образом, с точки зрения реализации новая атака отличается от атаки, описанной в работе [9], вычислением подходящих дробей к лучшему приближению дроби $\frac{k}{d}$, а именно, к числу $\frac{e}{N+1-2 N^{0.5}}$ вместо $\frac{e}{N}$. Кроме того, в новой атаке в качестве отправной точки для перебора значений $r$ и $s$ выбирается подходящая дробь, которая тем ближе к числу $\frac{e}{N+1-2 N^{0.5}}$, чем ближе простые $p$ и $q$. Например, если известно, что разность $p-q<N^{\beta}$, то нужно зафиксировать параметр $\lambda=0.75-\beta$ и найти номер последней нечетной подходящей дроби к числу $\frac{e}{N+1-2 N^{0.5}}$, для которой выполняется неравенство $\frac{P_{m}}{Q_{m}}-\frac{e}{N+1-2 N^{0.5}}>\frac{N^{1-2 \lambda}+4}{3 N}$. Далее секретную экспоненту $d$ необходимо искать среди линейных комбинаций $r Q_{m+1}+s Q_{m}, r Q_{m+2}-s Q_{m+1}$ и $r Q_{m+3}+s Q_{m+2}$ с помощью перебора по $r$ и $s$ с применением метода «встреча посередине».

\section{5. Эксперименты}

Наиболее трудоемкий этап атаки Дюжелла состоит в поиске чисел $r$ и $s$ из интервала $0 \leq r, s<4 D$, удовлетворяющих соотношению $a^{r} \equiv$ $\equiv 2 b^{s}(\bmod N)$ для фиксированных целых $a$ и $b$. Здесь $D$ - параметр метода, выбираемый в зависимости от имеющихся вычислительных и емкостных ресурсов и предполагаемого размера секретной экспоненты. 
В работе [9] Дюжелла предложил использовать метод «встреча посередине» для опробования пар $r$, $s$. Создается список длины $4 D$, coстоящий из пар $\left(r, h\left(a^{r} \bmod N\right)\right)$, где $h(x)$ - некоторая хэш-функция. Данный список сортируется по второй координате за время $O(D \ln D)$. Среди вторых координат списка для каждого опробуемого $s$ бинарным поиском ищется хэш-значение $h\left(2 b^{s} \bmod N\right)$ за время $O(\ln D)$. В худшем случае нужно перебрать $4 D$ различных чисел $s$ за время $O(D \ln D)$. В итоге трудоемкость метода составляет $O(D \ln D)$ с затратами памяти $O(D)$. При $D=2^{30}$ необходимо хранить $4 \cdot 2^{30}$ записей длиной 12 байтов $\left(\log _{2} 4 D\right.$ битов для числа $r$ и $2 \log _{2} 4 D$ для хэш-значения $\left.h\left(a^{r} \bmod N\right)\right)$, всего необходимо 48 Гб памяти. Можно сократить объем памяти, храня только 4 байта хэш-значений $h\left(a^{r} \bmod N\right)$. В этом случае, согласно парадоксу дней рождений [4], будут возникать совпадения вторых координат в списке, которые необходимо дополнительно проверять.

Вместо сортировки и поиска можно использовать хэш-таблицы. В этом случае нужно разместить $4 D$ четырехбайтовых чисел $r$ по адресам $h\left(a^{r} \bmod N\right)$. Хеш-функция $h(x)$ выбирается исходя из имеющихся объемов памяти. В условиях ограничения на память неизбежно возникают коллизии, которые разрешаются известными способами. При использовании хэштаблиц среднее время поиска и вставки равно $O(1)$, поэтому общая трудоемкость метода Дюжелла будет составлять $O(D)$ вместо $O(D \ln D)$ операций для поиска в сортированном списке. Для эффективной реализации хэш-таблиц нужно, чтобы ячеек памяти было больше, чем размещаемых элементов. Отсюда следует, что метод Дюжелла можно ускорить с увеличением расхода памяти. В условиях жестких ограничений на объем используемой памяти эффективность хэш-таблиц не очевидна. Для проведения экспериментов был реализован поиск в отсортированном списке.

Атака Дюжелла и новая атака были реализованы на языке C++ с помощью пакетов GMP [11] и MPFR [12]. Эти программы позволили провести эксперименты, результаты которых отражены в таблице 1. Эксперименты проводились на процессорe Intel Xeon с тактовой частотой 2.66 Ггц. Целью экспериментов было установить практические границы атаки, которые в данном случае определяются объемом расходуемой памяти. При этом не ставилась задача ускорения данных методов.

Из таблицы 1 видно, что если у 512-битовых простых чисел $p$ и $q$ совпадает около 60 старших битов, то множество слабых (малых) секретных экспонент $d$ существенно расширяется. При приближении разности $p-q$ к величине $N^{0.25}$ слабыми становятся все секретные экспоненты $d$, меньшие 
Таблица 1. Эксперименты с 1024-битовым модулем

\begin{tabular}{|c|c|c|c|c|c|}
\hline Атака & $p-q$, биты & $D$ & $d$, биты & Время, с & Память, Мб \\
\hline & & $10^{3}$ & 266 & 0.2 & $<1$ \\
Атака Дюжелла & \multirow{3}{*}{511} & $10^{6}$ & 276 & 135 & 48 \\
& & $10^{7}$ & 280 & 1500 & 480 \\
& & $10^{8}$ & 283 & 17200 & 4800 \\
\hline Новая атака & 507 & $10^{9}$ & 286 & 193000 & 48000 \\
\hline Атака де Вегера & 450 & 1 & 318 & 0.2 & 48000 \\
\hline & & $10^{3}$ & 328 & 0.2 & $<1$ \\
Новая атака & 450 & $10^{6}$ & 338 & 135 & 48 \\
& & $10^{7}$ & 341 & 1500 & 480 \\
& & $10^{8}$ & 344 & 17200 & 4800 \\
& & $10^{9}$ & 347 & 193000 & 48000 \\
\hline Атака де Вегера & 276 & 1 & 492 & 0.2 & $<1$ \\
\hline & & $10^{3}$ & 502 & 0.2 & $<1$ \\
& & $10^{6}$ & 512 & 135 & 48 \\
Новая атака & 276 & $10^{7}$ & 515 & 1500 & 480 \\
& & $10^{8}$ & 518 & 17200 & 4800 \\
& & $10^{9}$ & 521 & 193000 & 48000 \\
\hline
\end{tabular}

$N^{0.5}$. Значения $p-q<N^{0.25}$ не рассматривались, так как в этих случаях эффективно срабатывает метод факторизации Ферма.

Важно отметить, что проведенные эксперименты согласуются с экспериментами Дюжелла [9], в которых установлено, что на практике в $98 \%$ случаев числа $r$ и $s$ не превосходят $4 D$.

\section{6. Сравнение с обобщением атаки Боне и Дюрфи}

Де Вегер помимо обобщения атаки Винера предложил модификацию атаки Боне и Дюрфи на случай, когда $p$ и $q$ близки. В работе [10] показано, что секретная экспонента $d=N^{\delta}$ является нестойкой, если разность $p-q=N^{\beta}$ настолько мала, что выполняются неравенства $2-4 \beta<\delta<$ $<1-\sqrt{2 \beta-\frac{1}{2}}$ или $\delta<\frac{1}{6}(4 \beta+5)-\frac{1}{3} \sqrt{(4 \beta+5)(4 \beta-1)}$. При выполнении данных условий теоретически атака будет работать за полиномиальное время. На практике же границы атак на решетках далеки от теоретических. Де Вегер привел один пример практической реализации своей атаки. Зафиксировав 
$\beta=0.292$, он восстановил секретную экспоненту с $\delta=0.558$. Теоретически же для выбранного $\beta$ слабыми являются все экспоненты с величиной $\delta<0.71$. Для исходной атаки Боне-Дюрфи теоретическая граница равна $\delta=0.292$, хотя известные практические реализации работают при $\delta \leq 0.274$ (см., например [3]). На основании этих данных можно сделать предположение, что зазор между теоретическими и практическими границами атак составляет по меньшей мере величину $\Delta=\delta_{\text {теор }}-\delta_{\text {прак }}=0.018$.

Обобщение метода Дюжелла срабатывает при $d<\frac{D N^{0.75}}{p-q}$. В обозначениях $\delta=\log _{N} d$ и $\beta=\log _{N}(p-q)$ данное условие записывается как $\delta<0.75+\frac{\log _{2} D}{\log _{2} N}-\beta$. Эксперименты показывают (см. таблицу 1), что для 1024-битового модуля атака срабатывает при $D=30$. Отсюда следует, что слабые относительно данной атаки числа $\delta$ определяются неравенством $\delta<0.779-\beta$.

На рисунке 1 построены графики предполагаемых практических границ атаки Боне-Дюрфи и ее обобщения в сравнении с практическими границами атак Дюжелла и ее обобщением на интервале $0.485<\beta<0.5$. Пунктиром нарисованы графики для атаки Боне-Дюрфи (серая прямая $\delta=0.274$ ) и ее обобщения де Вегером (черная кривая $\delta=0.982-\sqrt{2 \beta-0.5}$ ). Сплошной линией нарисованы графики атак Дюжелла (серая прямая $\delta=0.279$ ) и ее обобщения (черная прямая $\delta=0.779-\beta$ ).

Из графиков видно, что предложенная в данной работе атака с практической точки зрения расширяет множество слабых ключей RSA

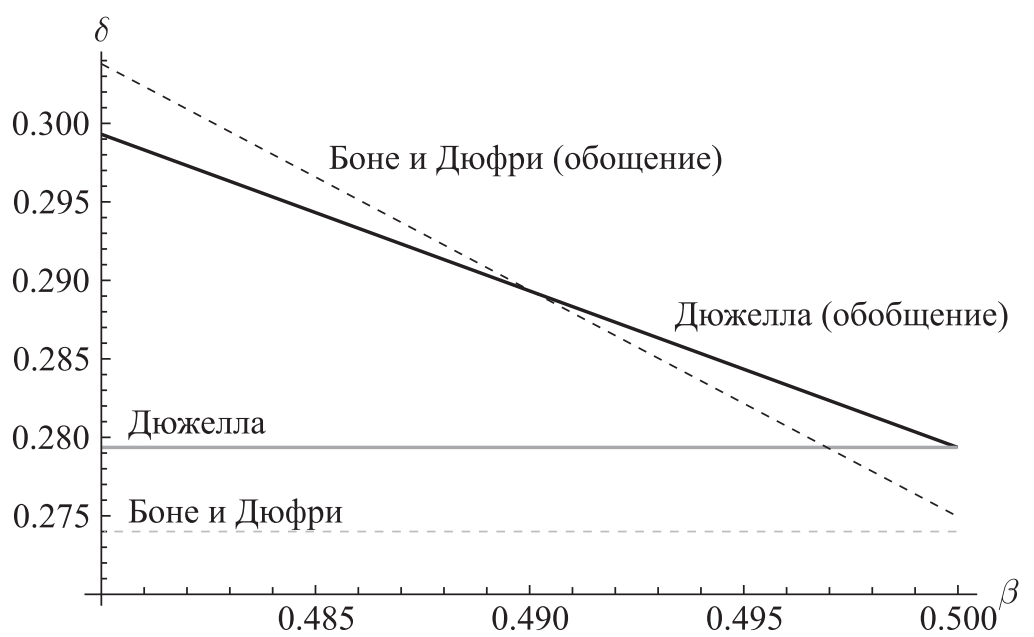

Рис. 1. Графики границ атак для 1024-битового модуля 
с 1024-битовым модулем и малой секретной экспонентой, если разность простых сомножителей лежит в интервале $N^{0.490}<p-q<N^{0.496}$. Например, если у 1024-битового модуля $N$ простые сомножители $p$ и $q$ совпадают в пяти старших битах, то новый метод позволяет атаковать 291-битовую экспоненту (см. таблицу 1), что по крайней мере на 2 бита больше предполагаемой практической границы обобщенной атаки Боне-Дюрфи.

\section{Список литературы}

1. Бухштаб А. А. Теория чисел. - М.: Просвещение, 1966.

2. Хинчин А. Я. Цепные дроби. - Л.: Физматгиз, 1961.

3. Hinek M. J. Cryptanalysis of RSA and Its Variants // CRC Press. - 2009.

4. Погорелов Б.А., Сачков В.Н. Словарь криптографических терминов. М.: МЦНМО, 2006.

5. Boneh D., Durfee G. Cryptanalysis of RSA with private key $d$ less than $N^{0.292} / /$ Advances in Cryptology - Proceedings of Eurocrypt'99, LNCS. 1999. - V. 1952. - P. 1-11.

6. Wiener M.J. Cryptanalysis of short RSA secret exponents // IEEE Trans. Inform. Theory. - 1990. - V. 36. - P. 553-558.

7. Verheul E.R., van Tilborg H.C.A. Cryptanalysis of 'less short' RSA secret exponents // Appl. Algebra Eng. Comm. Computing. - 1997. - V. 8. - P. 425435.

8. Dujella A. Continued fractions and RSA with small secret exponent // Tatra Mt. Math. Publ. - 2004. - V. 29. - P. 101-112.

9. Dujella A. A variant of Wiener's attack on RSA, Computing. - 2009. V. 85. - P. 77-83.

10. Weger B. Cryptanalysis of RSA with small prime difference // Appl. Algebra Eng. Comm. Computing. - 2002. - V. 13. - P. 17-28.

11. The GNU Multiple Precision Arithmetic Library // http://gmplib.org/.

12. The Multiple Precision Floating-Point Reliable Library // http: //www.mpfr.org/. 